\title{
Determinantes Psicológicos e Sociais relacionados ao desenvolvimento dos Transtornos Mentais no Puerpério: Uma revisão integrativa
}

\author{
Psychological and Social Determinants related to the Development of Mental Disorders in \\ Puerperium: A integrative review \\ Determinantes Psicológicos y Sociales relacionados con el Desarrolo de dos Trastornos Mentales \\ no Puerperales: Una revisíon integradora
}

\author{
Ântela Márcia Teles Queiroz \\ ORCID: https://orcid.org/0000-0003-2974-0515 \\ Centro Universitário Santo Agostinho, Brasil \\ E-mail: queirozantela@gmail.com \\ Luana Azevedo de Freitas \\ ORCID: https://orcid.org/0000-0001-5806-6115 \\ Centro Universitário Santo Agostinho, Brasil \\ E-mail: azevedoluana@outlook.com \\ Liana Dantas da Costa e Silva Barbosa \\ ORCID: https://orcid.org/0000-0002-8191-102X \\ Centro Universitário Santo Agostinho, Brasil \\ E-mail: dantasliana@bol.com.br
}

\begin{abstract}
Resumo
Compreender as evidências científicas acerca dos determinantes psicológicos e sociais relacionados ao surgimento de transtornos mentais no puerpério. O presente estudo foi desenvolvido através de uma revisão integrativa de caráter exploratória, através das bases de dados indexadas: LILACS (Literatura Latino-Americana e do Caribe em Ciências da Saúde) e MEDLINE (Medical Literature Analysis and Retrieval System Online). A partir do estudo foi evidenciado que as mudanças psicológicas e os fatores sociais na vida das mulheres podem desencadear problemas psicossociais ocasionando sérios transtornos mentais. É necessário o acompanhamento afetivo e profissional desde o início da vida gestacional, ao pós-parto e puerpério, a fim de prevenir danos à saúde física e mental da mulher. Portanto, é importante conhecer todos os fatores sociais, psicológicos dessas mulheres a fim de realizar o planejamento, apoio e a assistência.

Palavras-chave: Puerpério; Depressão pós-parto; Estresse pós-traumático; Ansiedade; Assistência de Enfermagem.
\end{abstract}

\begin{abstract}
Understand the scientific evidence about psychological and social determinants related to the emergence of mental disorders in the puerperium. The present study was developed through an integrative review of an exploratory caracter, the databases used in this research are indexed: LILACS (Latin American and Caribbean Literature in Health Sciences) and MEDLINE (Medical Literature Analyses and Retrieval System Online). During the research it was evidence that psychological changes and social factors in the lives of women can trigger psychosocial problems causing serious mental disorders. Affective and professional monitoring must be necessary from the beginning of gestational life, to the postpartum and puerperium period, in order to prevent damage to the woman's physical and mental health. Therefore, it is important to know all the social and psychological factors of these women in order to carry out planning, support and assistance.
\end{abstract}

Keywords: Puerperium; Baby blues; Post-traumatic stress disorder; Anxiety; Nursing assistance.

\section{Resumen}

Comprenderla evidencia científica sobre los determinantes psicológicos y sociales relacionados con la aparición de trastornos mentales en el puerperio. El presente estudio se desarrolló mediante una revisión integradora de carácter exploratorio, indexada como bases de datos utilizadas en esta investigación: LILACS (Literatura Latinoamericana y Caribeña Ciencias de la Salud) y MEDLINE (Análisis de la Literatura Médica) y el sistema de recuperación en línea). Durante la investigación se demostró que los cambios psicológicos y los factores sociales en la vida de las mujeres pueden desencadenar problemas psicosociales que provocan graves trastornos mentales. El seguimiento afectivo y profesional debe ser necesario desde el inicio de la vida gestacional, hasta el posparto y puerperio, para prevenir daños a la salud física y mental de la mujer. Por tanto, es importante conocer todos los factores sociales y psicológicos de estas mujeres para poder realizar la planificación, el acompañamiento y la asistencia.

Palabras clave: Puerperio; Baby blues; Trastorno de estrés postraumático; Ansiedad; Asistencia de enfermería. 


\section{Introdução}

O desenvolvimento de problemas psicológicos durante a gravidez até o puerpério são condições que podem influenciar em situações irreversíveis na fase gestacional. Esses problemas, quando não diagnosticados e tratados prematuramente podem interferir futuramente de forma negativa na vida da mulher e do bebê (Aleixo \& Gonçalves,2017). No ciclo gravídico puerperal, o puerpério é a fase de mais ampla vulnerabilidade psíquica, onde ocorre o desencadeamento de variações psicoemocionais e corporais, somada também com as condições sociais, poderá permitir o surgimento de transtornos mentais no puerpério (Maciel et al., 2019).

Durante toda a gestação até o período do puerpério, ocorrem fortes mudanças na vida das mulheres, sendo essas culturais, sociais, fisiológicas e principalmente emocionais, tornando os sentimentos mais intensos nos primeiros dias após o parto e desafiador no processo de se tornar mãe. Nesse período, a mulher necessita de atenção devido às intensas alterações hormonais, grandes cobranças socioculturais, familiares e pessoais, situações essas consideradas como os principais fatores geradores de estresse, desgaste físico e emocional intensos na mulher, influenciando assim sua saúde mental e tornando-a mais vulnerável ao estresse e ao aparecimento de transtornos mentais (Melo et al., 2018).

No puerpério, além das mudanças nos níveis hormonais e alterações biológicas, também se constata mudanças psicológicas e sociais que exigem da mulher uma reorganização social, além de adaptações ao seu novo papel aumentando sua responsabilidade por cuidar de uma pessoa indefesa, fazendo privações do sono e se isolando ou reduzindo consideravelmente sua participação social. Além disso, a mulher precisa se reestruturar da sua imagem corporal, da sua sexualidade e da sua identidade feminina (Cantilino et al., 2010).

Nessa perspectiva, essa nova relação pouco estruturada entre a mãe e o bebê, poderá provocar nas puérperas, sensações conturbadas no relacionamento de mãe-filho, onde podem apresentar alguns sinais relacionados aos transtornos mentais devido às suas fr---agilidades psíquicas enfrentadas nessa fase, que podem ser agravadas por fatores sociais, comportamentais e de relacionamentos (Maciel et al., 2019). Devido ao puerpério ser um período de muita vulnerabilidade, as puérperas manifestam sentimento de tristeza, ansiedade, fadiga, diminuição da concentração, preocupação, irritabilidade e insônia, podendo desencadear transtornos mentais comuns. A baixa renda e a obesidade também são fatores de probabilidade para o desencadeamento desses transtornos (Carvalho et al., 2019).

A insegurança e o medo são fatores que podem instalar sentimentos de angústia, preocupações e dúvidas, causando frustrações na mulher sobre sua visão à maternidade principalmente na hora de pôr em prática as obrigações exigidas pela maternagem. Sendo assim, todos esses determinantes podem gerar conflitos de pensamentos e atitudes, o desenvolvimento de transtornos mentais nessa fase (Maciel et al., 2019).

Um dos transtornos mentais relacionados à gravidez e à maternidade é a ansiedade e caracteriza-se por preocupações excessivas e irrealistas podendo ser compreendida através do emocional. É caracterizada na puérpera por um estado de insatisfação, insegurança, incerteza e medo da experiência desconhecida (Airosa \& Silva, 2013). O transtorno do estresse póstraumático (TEPT) também é um agravo mental que ocorre em resposta a eventos que se desenvolvem a partir da vivência de situações traumáticas relacionadas ao parto ou à saúde da criança em seus primeiros dias de vida. Os principais fatores de risco seriam as complicações maternas e neonatais, algum trauma antes ou durante a gestação, mesmo não sendo no ciclo gestacional (Henriques et al., 2015).

Assim como a ansiedade, durante a gravidez, $10 \%$ a 15\% das mulheres vivenciam sintomas de depressão leves e/ou moderados, apresentando sintomas semelhantes à de outros períodos, como a falta de apetite, energia e sentimento de culpa. Essas manifestações podem interferir no processo adequado de desenvolvimento fetal, provocar resultados desfavoráveis como um parto prematuro e baixo peso ao nascer, podendo persistir no período pós-parto comprometendo o comportamento parental, 
o relacionamento familiar, o processo de formação do vínculo entre mãe e filho e o desenvolvimento da criança (LIMA et al., 2017).

A depressão pós-parto (DPP) é o transtorno psiquiátrico mais incidente nas puérperas e tem prevalência entre 13\% a $19 \%$ em países desenvolvidos. Os principais fatores relacionados ao transtorno são: apoio familiar e social inexistentes ou inadequados, antecedentes psiquiátricos na mulher, ansiedade intensa, existência de episódios depressivos anteriores, infertilidade e sentimentos negativos em relação à gestação ou ao bebê (Abuchaim et al., 2016).

Outros fatores de riscos podem estar associados à DPP, como: gestante solteira, conflitos conjugais, histórico familiar de depressão, gravidez não programada, gestante com menos de 17 anos ou mais de 40 anos, uso de drogas ou álcool, tratamento ou hospitalização psiquiátrica anterior, histórico obstétrico de risco (aborto, prematuridade, placenta prévia, natimorto, más-formações), histórico familiar de outras doenças mentais (Almeida \& Arrais., 2016).

Dos sintomas mais comuns da DPP estão o desânimo persistente, sentimento de culpa, alterações do sono, ideias suicidas, temor de machucar o filho, redução do apetite e da libido, diminuição do nível de funcionamento mental e presença de ideias obsessiva (Corrêa \& Serralha, 2015).

O surgimento do humor depressivo faz com que a mulher perca o prazer e o interesse nas atividades que realizava, com a alteração do sono pode apresentar insônias ou sono excessivo; apresentar sentimento de desamparo e desesperança, falta de energia, falta de motivação, sensação de ser incapaz de lidar com as mudanças, sentimentos confusos em relação ao filho, negligência de cuidados ao filho e diminuição no período de amamentação (Schwengber \& Piccinini, 2005).

Esses sintomas mencionados podem prejudicar o desenvolvimento neurobiológico e psicológico da criança, que necessita do acolhimento e cuidados da mãe para seu desenvolvimento saudável, e também a própria saúde da mulher. A partir disso, o aconselhamento e o esclarecimento adequados à gestante ou puérpera podem servir de ajuda imediata e auxiliar na prevenção do aparecimento de transtornos psicológicos (Corrêa \& Serralha, 2015).

Diante da gravidade destes transtornos é observada a necessidade de adaptação que a puérpera precisa enfrentar a partir de recursos que tentem preveni-los, como o pré-natal psicológico, que se trata de um novo conceito em atendimento perinatal, voltado para uma maior humanização do processo gestacional, do parto e de construção da parentalidade (Almeida \& Arrais, 2016).

As ações de saúde desenvolvidas no pré-natal devem dar cobertura a todas as gestantes, assegurando o acompanhamento e a continuidade no atendimento e avaliação. Esse atendimento tem como objetivo a prevenção, a identificação e correção das intercorrências maternas e fetais, bem como educar as gestantes a respeito da gravidez, parto, puerpério e cuidados com o recém-nascido (Maeda et al., 2014).

Dessa forma, o acolhimento é um aspecto essencial da política de humanização, que deve ser realizado com responsabilidade desde a chegada da paciente na unidade de saúde, em que os profissionais devem dar atenção às demandas e queixas relatadas pela gestante, permitindo que ela expresse suas preocupações, angústias, garantindo atenção resolutiva e articulação com os outros serviços de saúde para a continuidade da assistência quando necessária. A intervenção psicológica também é importante para buscar conter as angústias dos familiares na medida em que se explicita a importância da criação de um espaço seguro à relação mãe-bebê (Souza \& Acácio, 2019).

A preocupação e apoio dos profissionais de saúde aos pacientes e aos seus familiares permite que estes sintam-se mais firmes e confiantes na expressão de seus sentimentos, garantindo uma acolhida humanizada (Guedes et al., 2003).O enfermeiro obstetra tem muito a contribuir ao estudar o puerpério, reconhecendo a individualidade de cada puérpera quanto aos seus sentimentos e expectativas nesse momento de vida, podendo a mulher receber a ajuda que precisa em seu meio familiar e ter adequada assistência profissional (Soler, 2014). 
Logo, o estudo tem como objetivo principal compreender e analisar as evidências científicas acerca dos determinantes psicológicos e sociais relacionados ao surgimento de transtornos mentais no puerpério e apresentar estratégias da assistência de enfermagem quanto a prevenção e enfrentamento de transtornos mentais surgidos no puerpério.

Nesse período puerperal a mulher deve-se adaptar ao novo processo, o de se tornar mãe. É o momento em que estará em recuperação e ao mesmo tempo de reorganização do seu cotidiano na inclusão do bebê em sua vida, sendo assim muitas mulheres se veem incapazes, despreparadas, sem ânimo para lidar com essa nova experiência, com isso, podendo desencadear conflitos internos e possibilitar o desenvolvimento de problemas psicológicos.

Em vista disso, é importante conhecer todas essas mudanças fisiológicas, psicossociais e fatores de risco desde o início da gestação até o pós-parto para o desencadeamento desses problemas psicológicos. Sobretudo o conhecimento adquirido facilitará então o planejamento, as ações preventivas, as intervenções precoces, o apoio social e principalmente o apoio da enfermagem obstétrica ligada às estratégias psicológicas.

\section{Metodologia}

Foi realizado um estudo de revisão da literatura do tipo revisão integrativa, qualitativa de caráter exploratório, com base na seguinte questão de pesquisa: Quais determinantes psicológicos e sociais estão relacionados ao surgimento de transtornos mentais no puerpério?

A elaboração da pergunta norteadora, busca ou amostragem na literatura, coleta de dados, análise crítica dos estudos incluídos, discussão dos resultados e a apresentação da revisão integrativa características do estudo do tipo revisão integrativa, de forma que essas seis etapas influenciam de forma conjuntada ao um único objetivo desse tipo de estudo, onde se é dada como método facilitário nas buscas de pesquisas bibliográficas (Souza, Silva \& Carvalho, 2010).

A coleta dos estudos para a pesquisa foi realizada nos meses de fevereiro a abril de 2021 por meio de busca online em bases de dados científicos da saúde. A busca ocorreu no banco de dados da Biblioteca Virtual em Saúde (BVS), onde estão indexadas as bases de dados como Literatura Latino-americana e do Caribe em Ciências as Saúde (LILACS) e PUBMED. Como descritores foram utilizadas as palavras cadastradas nos Descritores em Ciências de Saúde (DeCS): Puerpério, Depressão pós-parto, Estresse pós-traumático, Ansiedade, assistência de Enfermagem.

Após a busca inicial dos estudos por meio dos descritores escolhidos foram usados como critérios de inclusão da pesquisa: apenas artigos disponíveis na íntegra, publicados nos idiomas em inglês, português e espanhol, publicados entre janeiro de 2010 à janeiro de 2020 totalizando o período de 10 anos, com temas que tenham relevância para a temática proposta. Os critérios de exclusão foram as classificações de materiais onde os textos estejam repetidos nas bases de dados e que não apresentavam relevância para o tema do estudo.

Após a seleção dos estudos por meio do processo metodológico descrito acima, os artigos selecionados foram submetidos à análise por meio de quadros e figuras, a fim de traçar o perfil das publicações, onde seguirá primeiramente a estrutura organizacional dos dados. É de afirmação que seguramente a revisão dos dados a de ser feita e analisada pelos pesquisadores, sendo um deles o orientador da pesquisa, onde proporcionará o cruzamento de informações de evidências sobre o assunto da qual serão discutidos em embasamento a da literatura atual. 
Figura 1. Descrição do percurso para a seleção dos estudos.

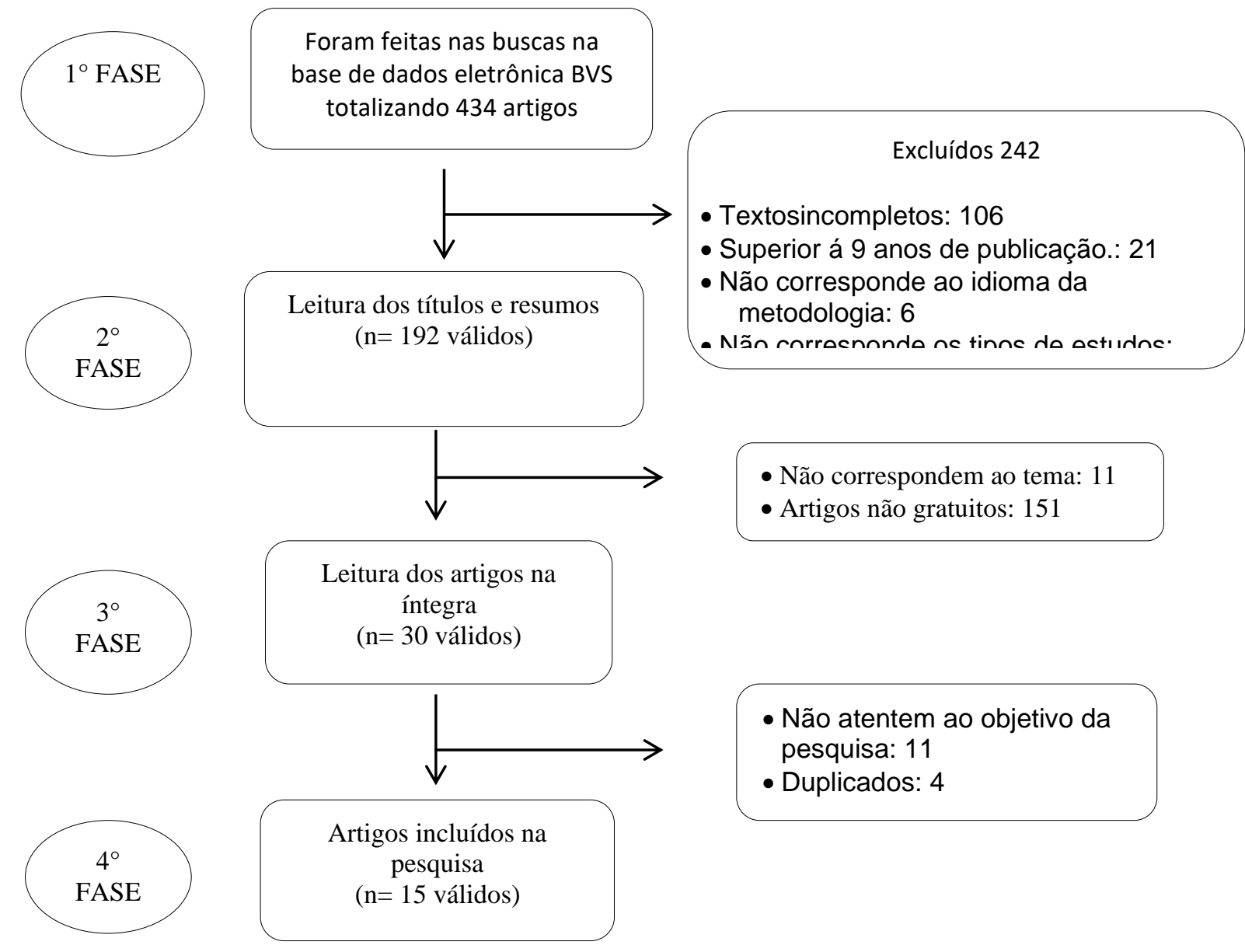

Fonte: Autores.

\section{Resultados e Discussão}

A pesquisa inicial, realizada nas bases de dados eletrônicas, por meio da combinação dos descritores "Período pósparto", "depressão pós-parto", "transtornos de estresse pós-traumáticos" e "Ansiedade” em associações com os operadores booleanos “AND" e “OR", identificou 434 artigos que constam na BVS. Totalizou-se 413 na MEDLINE e 21 na LILACS. Após Teste de Relevância 1, foram selecionados 30 artigos, já após análise a partir do Teste de Relevância 2 foram selecionados 15 artigos (Tabela1).

Tabela 1. Distribuição das referências obtidas nas bases de dados MEDLINE e LILACS seguindo os descritores estabelecidos. Teresina, 2021.

\begin{tabular}{lccc}
\hline Base de dados & Descritores cruzados & $\begin{array}{c}\text { Referências } \\
\text { obtidas }\end{array}$ & Referências selecionadas \\
\hline Período pós-parto & \\
AND & 309 & 7 \\
DEDLINE & Depressão pós-parto & \\
OR & \\
& Transtornos de estresse pós- \\
& traumático & \\
\hline
\end{tabular}




\begin{tabular}{|c|c|c|c|}
\hline \multirow{5}{*}{ MEDLINE } & Período pós-parto & \multirow{5}{*}{104} & \multirow{5}{*}{7} \\
\hline & AND & & \\
\hline & $\begin{array}{c}\text { Transtornos de estresse pós- } \\
\text { traumáticos }\end{array}$ & & \\
\hline & AND & & \\
\hline & Ansiedade & & \\
\hline \multirow{6}{*}{ LILACS } & Período pós-parto & \multirow{6}{*}{16} & \multirow{6}{*}{1} \\
\hline & AND & & \\
\hline & Depressão pós-parto & & \\
\hline & OR & & \\
\hline & Transtornos de estresse pós- & & \\
\hline & traumático & & \\
\hline \multirow{6}{*}{ LILACS } & Período pós-parto & \multirow{6}{*}{5} & \multirow{6}{*}{0} \\
\hline & AND & & \\
\hline & Transtornos de estresse pós- & & \\
\hline & . & & \\
\hline & OR & & \\
\hline & Ansiedade & & \\
\hline TOTAL & & 434 & 15 \\
\hline
\end{tabular}

Fonte: Biblioteca Virtual em Saúde.

Tabela 2. Número de estudos selecionados por Teste de Relevância.

\begin{tabular}{|c|c|c|c|}
\hline & Busca Inicial & Teste de Relevância 1 & Teste de Relevância 2 \\
\hline MEDLINE & 413 & 19 & 14 \\
\hline LILACS & 21 & 11 & 1 \\
\hline TOTAL & 434 & 30 & 15 \\
\hline
\end{tabular}

Fonte: Biblioteca Virtual em Saúde.

O Quadro 1, exibe os estudos que compuseram esta revisão integrativa, no que se refere ao título, à base de dados, ao tipo de estudo/abordagem, ao ano, idioma de publicação, objetivo da pesquisa e aos dados evidenciados conclusivos. 
Quadro 1. Características gerais dos trabalhos utilizados na presente revisão.

\begin{tabular}{|c|c|c|c|c|c|c|}
\hline $\mathbf{N}^{\circ}$ & AUTORES & TÍTULO & $\begin{array}{l}\text { BASE DE } \\
\text { DADOS }\end{array}$ & ABORDAGEM & ANO (PAÍS) & OBJETIVO \\
\hline I & $\begin{array}{l}\text { (WITTEVEEN et } \\
\text { al., 2020) }\end{array}$ & $\begin{array}{l}\text { The oxytocinergic system in } \\
\text { PTDS } \\
\text { following traumatic } \\
\text { cheldbirth: endogenous and } \\
\text { exogenous oxytocin in the } \\
\text { perinatum period. }\end{array}$ & MEDLINE & Narrativa & 2020 (Inglês) & $\begin{array}{l}\text { Avaliar as alterações } \\
\text { biológicas no sistema } \\
\text { oxitocinérgico no } \\
\text { comportamento e afeto } \\
\text { materno pós-parto. }\end{array}$ \\
\hline II & $\begin{array}{ll}(\text { BECK } & \& \\
\text { CASAVANT } & \text { et } \\
\text { al., 2019) } & \end{array}$ & $\begin{array}{l}\text { Synthesis of mixed research } \\
\text { on posttraumatic stress } \\
\text { related to-traumatic birth. }\end{array}$ & MEDLINE & $\begin{array}{l}\text { Qualitativa } \\
\text { quantitativa }\end{array}$ & 2019 (Inglês) & $\begin{array}{l}\text { Sintetizar os } \\
\text { pesquisas } \\
\text { (quantitativas e qualitativas) } \\
\text { sobre o estresse pós } \\
\text { traumático em mulheres } \\
\text { experientes em partos } \\
\text { traumáticos. }\end{array}$ \\
\hline III & $\begin{array}{l}\text { (KJELDGAARD } \\
\text { et al., 2018) }\end{array}$ & $\begin{array}{l}\text { The association between the } \\
\text { degree of nausea in regnancy } \\
\text { and subsequent posttraumatic } \\
\text { stress }\end{array}$ & MEDLINE & Coorte & $\begin{array}{l}2018 \\
\text { (Inglês) }\end{array}$ & $\begin{array}{l}\text { Avaliar se a hiperêmese } \\
\text { gravídica (HG) } \\
\text { aumenta o risco de doença } \\
\text { relacionada ao parto com } \\
\text { sintomas de estresse pós- } \\
\text { traumático (PTSS). }\end{array}$ \\
\hline IV & $\begin{array}{l}\text { (AHMED et al., } \\
\text { 2017) }\end{array}$ & $\begin{array}{l}\text { aternal depression in syrian } \\
\text { refugee women recently } \\
\text { moved to canada: a } \\
\text { preliminary study. }\end{array}$ & MEDLINE & Qualitativo & $\begin{array}{l}2017 \\
\text { (Inglês) }\end{array}$ & $\begin{array}{l}\text { Fornecer um contexto rico } \\
\text { em descrições das } \\
\text { configurações, } \\
\text { participantes bem como } \\
\text { resultados da análise } \\
\text { qualitativa. }\end{array}$ \\
\hline V & $\begin{array}{l}\text { (OSTACOLI et al., } \\
2020)\end{array}$ & $\begin{array}{l}\text { Psychosocial factors } \\
\text { associated with postpartum } \\
\text { psychological } \\
\text { distress during the Covid-19 } \\
\text { pandemic: a cross-sectional } \\
\text { study. }\end{array}$ & MEDLINE & Coorte & $\begin{array}{l}2020 \\
\text { (Inglês) }\end{array}$ & $\begin{array}{l}\text { Avaliar os fatores associados } \\
\text { de } \\
\text { depressão pós-parto e PTSS } \\
\text { durante a pandemia de } \\
\text { Covid-19. }\end{array}$ \\
\hline VI & $\begin{array}{l}\text { (AHMED et al., } \\
2019)\end{array}$ & $\begin{array}{l}\text { Trajectories of maternal } \\
\text { depressive and anxiety } \\
\text { symptoms from pregnancy to } \\
\text { five years postpartum and } \\
\text { their preonatalpredictons. }\end{array}$ & MEDLINE & Sistemática & 2019 (Inglês) & 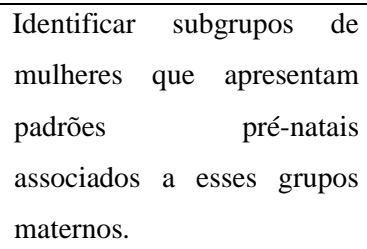 \\
\hline VII & $\begin{array}{l}\text { (BENTON et al., } \\
\text { 2019) }\end{array}$ & $\begin{array}{l}\text { Women's Psychosocial } \\
\text { outcomes following an } \\
\text { emergency caesarean section: } \\
\text { a systematic literature review. }\end{array}$ & MEDLINE & Sistemática & 2019 (Inglês) & $\begin{array}{l}\text { Identificar, comparar e } \\
\text { examinar as evidências que } \\
\text { cercam } \\
\text { psicossociais da cesariana de } \\
\text { emergência (EmCS) em } \\
\text { mulheres em todo o mundo. }\end{array}$ \\
\hline VIII & $\begin{array}{l}\text { (ANGELINI et al, } \\
2018 \text { ) }\end{array}$ & $\begin{array}{l}\text { Post-traumatic stress } \\
\text { disordem and severe maternal } \\
\text { morbidity: is there an }\end{array}$ & MEDLINE & Coorte & $\begin{array}{l}2018 \\
\text { (Inglês) }\end{array}$ & $\begin{array}{l}\text { Avaliar a ocorrência de } \\
\text { transtorno de estresse } \\
\text { pós-traumático entre }\end{array}$ \\
\hline
\end{tabular}




\begin{tabular}{|c|c|c|c|c|c|c|}
\hline & & association? & & & & $\begin{array}{l}\text { mulheres que vivenciam um } \\
\text { quadro severo de evento de } \\
\text { mobilidade materna e fatores } \\
\text { associados em comparação } \\
\text { com aqueles sem morbidade } \\
\text { materna. }\end{array}$ \\
\hline IX & $\begin{array}{l}\text { (SENTILHES et } \\
\text { al., 2017) }\end{array}$ & $\begin{array}{l}\text { Risk factors for chronic port- } \\
\text { traumatic stress disorder } \\
\text { development one year after } \\
\text { vaginal delivery: a } \\
\text { prospective, observational } \\
\text { study. }\end{array}$ & MEDLINE & Coorte & $\begin{array}{l}2017 \\
\text { (Inglês) }\end{array}$ & $\begin{array}{l}\text { Avaliar a prevalência de } \\
\text { transtorno de estresse pós } \\
\text { traumático (TEPT) após o } \\
\text { parto }\end{array}$ \\
\hline$X$ & $\begin{array}{ll}\text { (KING, } & \text { MCHARG } \\
\& & \text { HORSCH, } \\
2017) & \end{array}$ & $\begin{array}{l}\text { Testing a cognitive model to } \\
\text { predict posttraumatic stress } \\
\text { disorder following childbirth. }\end{array}$ & MEDLINE & Qualitativo & $\begin{array}{l}2017 \\
\text { (Inglês) }\end{array}$ & $\begin{array}{l}\text { Investigar se as variável } \\
\text { derivadas teoricamente no } \\
\text { modelo cognitivo explicam a } \\
\text { variância única em sintomas } \\
\text { de PTDS pós-natal quando } \\
\text { os principais fatores de } \\
\text { riscos demográficos, } \\
\text { obstétricos e clínicos são } \\
\text { controlados. }\end{array}$ \\
\hline XI & $\begin{array}{l}\text { (LOPEZ et al., } \\
2017)\end{array}$ & $\begin{array}{l}\text { Post-traumatic stress disorder } \\
\text { in patients delivering by } \\
\text { caesarean section and the } \\
\text { implication of anaesthesia: a } \\
\text { prospective cohort study. }\end{array}$ & MEDLINE & Coorte & $\begin{array}{l}2017 \\
\text { (Inglês) }\end{array}$ & $\begin{array}{l}\text { Avaliar os fatores associados } \\
\text { à anestesia } \\
\text { desenvolvimento de } \\
\text { agudo pós-parto. }\end{array}$ \\
\hline XII & $\begin{array}{l}\text { (CARROLL, } \\
\text { DARLY \& } \\
\text { BEGLEY, 2016) }\end{array}$ & $\begin{array}{l}\text { The prevalence of women's } \\
\text { emotional and psysical health } \\
\text { problems following a } \\
\text { postpartum haemorrhage: a } \\
\text { systematic review. }\end{array}$ & MEDLINE & Sistemática & $\begin{array}{l}2016 \\
\text { (Inglês) }\end{array}$ & $\begin{array}{l}\text { Determinar a prevalência } \\
\text { geral de problemas } \\
\text { emocionais e físicos em } \\
\text { problemas de saúde } \\
\text { experimentados por } \\
\text { mulheres após uma } \\
\text { hemorragia pós-parto. }\end{array}$ \\
\hline XIII & $\begin{array}{l}\text { (REDSHAW, } \\
\text { HENNEGAN \& } \\
\text { HENDERSON, } \\
\text { 2016) }\end{array}$ & $\begin{array}{l}\text { Impact of holding the baby } \\
\text { following stillbirth on } \\
\text { maternal mental hearth and } \\
\text { well-being: findings from a } \\
\text { national survey. }\end{array}$ & MEDLINE & Sistemática & $\begin{array}{l}2016 \\
\text { (Inglês) }\end{array}$ & $\begin{array}{l}\text { Comportar a saúde mental e } \\
\text { bem estar em } \\
3 \text { a } 9 \text { meses após o } \\
\text { nascimento de natimorto } \\
\text { entre mulheres que } \\
\text { seguraram ou não } \\
\text { seguraram o seu bebê, } \\
\text { ajustado para diferenças } \\
\text { demográficas e clinicas. }\end{array}$ \\
\hline XIV & $\begin{array}{l}\text { (TORCHALLA et } \\
\text { al., 2014) }\end{array}$ & $\begin{array}{l}\text { Like a lots happened with my } \\
\text { whole childhood: violence, } \\
\text { trauma, and addiction in } \\
\text { pregnant and post partum } \\
\text { women from vancolver's }\end{array}$ & MEDLINE & Qualitativo & $\begin{array}{l}2014 \\
\text { (Inglês) }\end{array}$ & $\begin{array}{l}\text { Explorar temas e } \\
\text { perspectivas de trauma e } \\
\text { violência de gênero em } \\
\text { mulheres que viviam em um } \\
\text { bairro pobre e lutavam }\end{array}$ \\
\hline
\end{tabular}




\begin{tabular}{|c|c|c|c|c|c|c|}
\hline & & downtown eastside. & & & & $\begin{array}{l}\text { contra o uso de substâncias } \\
\text { durante a gravidez e o início } \\
\text { da maternidade. }\end{array}$ \\
\hline XV & $\begin{array}{l}\text { (HENRIQUES et } \\
\text { al., 2015) }\end{array}$ & $\begin{array}{l}\text { Transtorno de estresse pós- } \\
\text { traumático no puerpério em } \\
\text { uma maternidade de alto } \\
\text { risco fetal no município do } \\
\text { Rio de Janeiro, Brasil. }\end{array}$ & MEDLINE & Transversal & $\begin{array}{l}2015 \\
\text { (Português) }\end{array}$ & $\begin{array}{l}\text { Estimar a magnitude de } \\
\text { transtorno do estresse } \\
\text { pós-traumático (TEPT) } \\
\text { no puerpério em uma } \\
\text { maternidade de referência } \\
\text { para agravos perinatais e } \\
\text { identificar subgrupos } \\
\text { vulneráveis. }\end{array}$ \\
\hline XVI & $\begin{array}{l}\text { (CANTILINO, A } \\
\text { et al., 2010) }\end{array}$ & $\begin{array}{l}\text { Transtornos psiquiátricos no } \\
\text { pósparto. }\end{array}$ & LILACS & Bibliográfica & $\begin{array}{l}2010 \\
\text { (Português) }\end{array}$ & $\begin{array}{l}\text { Analisar os tipos de } \\
\text { transtornos } \\
\text { psiquiátricos no pós-parto. }\end{array}$ \\
\hline
\end{tabular}

Fonte: Autores.

O Quadro 2, apresenta os subtítulos utilizados na discussão dos artigos selecionados, para facilitar o entendimento e a explanação do conteúdo abaixo. O foco do estudo, envolverá três subtemas: Transtornos psiquiátricos no pós-parto; Motivos relacionados ao surgimento dos transtornos e Assistência de Enfermagem na prevenção e enfrentamento de transtornos mentais surgidos no puerpério.

Quadro 2. Distribuição dos artigos em Foco do estudo e Identificação dos Artigos.

\begin{tabular}{|c|c|}
\hline FOCO DO ESTUDO & $\begin{array}{c}\text { INDENTIFICAÇÃO DOS ARTIGOS POR AUTORES } \\
\text { Transtornos psiquiátricos no pós-parto }\end{array}$ \\
$\begin{array}{c}\text { (Beck \& Casavant, 2019); (Ahmed, Bowen \& Feng, 2017); } \\
\text { (Angelini et al., 2018); (Lopez et al., 2017); (Cantilino et al., } \\
\text { 2010); (Ahmed; Bowen; Feng \& Muhajarine, 2019). }\end{array}$ \\
\hline $\begin{array}{c}\text { (Witteveen et al., 2020); (King, Mcharg \& Horsch, 2017); } \\
\text { Motivos determinantes dos transtornos }\end{array}$ & $\begin{array}{r}\text { (Kjeldgaard et al., 2018); (Ostacoli et al., 2020); (Benton et al., } \\
\text { 2019); (Sentilhes et al., 2017); (Carroll, Darly \& Begley, } \\
\text { 2016); (Redshaw, Hennegan \& Henderson, 2016). }\end{array}$ \\
\hline $\begin{array}{c}\text { Assistência de Enfermagem na prevenção } \\
\text { e enfrentamento de transtornos mentais } \\
\text { surgidos no puerpério. }\end{array}$ & (Torchalla et al., 2014); (Henriques et al., 2015). \\
\hline
\end{tabular}

Fonte: Autores.

Diante dos achados nas publicações, o estudo permitiu fazer uma análise de algumas categorias temáticas que estão descritas abaixo:

\subsection{Transtornos psiquiátricos no pós-parto}

$\mathrm{Na}$ vida da mulher um dos eventos considerados mais desafiadores e de fortes emoções é o parto, pois nele a mesma experimenta várias emoções, principalmente o de alegria ao receber um novo filho, até mesmo algumas experimentando o medo pela vida e bem estar desse novo nascido e também os seus próprios, podendo ocasionar possíveis complicações psicológicas. Assim, ao vivenciarem eventos graves no período maternal, as mulheres necessitam e devem receber cuidados diferenciados das demais (Lopez et al., 2017). 
No puerpério, há muitas mudanças nos níveis hormonais dessas mulheres, principalmente relacionados ao sistema neurotransmissor, nos níveis de ocitocina e no eixo hipotálamo-hipófise adrenal. A transição para a maternidade também é muito marcante devido às alterações biológicas, psicológicas e sociais. A reorganização social e adaptação a esse novo papel é visto como necessário para que a mulher tenha mais responsabilidade, pois ela se tornará referência a uma pessoa indefesa e devido a isso sofrerá distúrbios de sono e se isolamento social (Cantilino et al., 2010).

A experiência de grande maioria das mulheres ter um bebê é de grande alegria, porém também é uma experiência estressante, com ansiedade, depressão e mau humor para 10-19\% das mulheres grávidas e 9-14\% das mulheres pós-natal em todo o mundo. Sabe-se que 15 a 57\% das mulheres, experimentam depressão durante a gestação ou até no pós-parto em países mais desenvolvidos (Ahmed, Bown \& Feng, 2017).

Para Cantilino et al. (2010), o período do pós-parto é o que possui maior vulnerabilidade para o surgimento dos transtornos psiquiátricos comparado a outros períodos, sendo os transtornos de estresse pós-traumático (TEPT), a depressão pós-parto (DPP), entre outros, os transtornos mais relacionados a esse período, mesmo que consideradas distintas atualmente nos sistemas classificatórios.

A mulher ao vivenciar o estresse pós-traumático relacionado a traumas na hora do parto, também traz vulnerabilidade a toda a sua unidade familiar. Ao estudar as previsões desse fato, há possibilidade de ajuda no desenvolvimento de rastreamento de mulheres que possuem esses riscos e fatores para o desencadeamento do estresse pós-traumático relacionado a esse trauma do nascimento. Além disso, as intervenções primárias também são necessárias para prevenir os traumas que essas mulheres podem vivenciar, (Beck \& Casavant, 2019).

Segundo Angelini et al. (2017), os transtornos de estresse pós-traumático (PTSD), poderá interferir negativamente na cognição infantil, no desenvolvimento, na linguagem e também afetar o relacionamento com o parceiro e a família principalmente, durante o parto ou período de pós-parto. O desencadeamento do PTSD surgirá após a exposição de traumas, provavelmente ocasionará danos à pessoa em se ao redor, tendo como respostas o intenso medo, o desamparo e impotência a situação e o horror.

Porém, o transtorno que causa mais prejuízos é a depressão pós-parto (DPP), principalmente na relação mãe-bebê e no seu desenvolvimento, os bebês exibem mais afeto negativo do que positivo quando as mães são deprimidas, possuem menor nível de atividade e menos vocalizações, distanciam o olhar, são mais aborrecidos, possuem expressões de tristeza e raiva e menos expressões de interesse e apresentam a aparência depressiva com poucos meses de idade (Cantilino et al., 2010).

Assim como a depressão, a ansiedade também possui uma prevalência de $30 \%$ em mulheres grávidas, isso seria por causa dos resultados negativos na saúde dessa mulher e o da sua família, possibilitando assim complicações no desenvolvimento da criança e psicológico quando não acompanhado corretamente, também afeta a saúde maternal, sendo porta de entrada para o declínio físico e cognitivo (Ahmed et al., 2019).

\subsection{Motivos determinantes dos transtornos}

Ser mulher já é um grande fator de risco para o desenvolvimento de sintomas, como estresse pós-traumático (TEPT) e os depressivos, atingindo geralmente o público adolescente e adultos. A vulnerabilidade dessas mulheres pode se ocasionar por causa dos fatores estressores, sendo eles: as crises de saúde e desastres naturais. A causa mais comum para as morbidades dessas mulheres pode acontecer devido os distúrbios mentais na gravidez, como a depressão que atinge $12 \%$ e a ansiedade $22 \%$ no fim da gestação. Além disso, o parto também pode ser uma experiência traumática, devido a complicações vivenciadas, o medo, a falta de apoio, entre outros (OSTACOLI et a., 2020).

Witteveen et al. (2020), relata que essas experiências de parto podem ser traumáticas, podendo originar o TEPT após o parto. Principalmente, as alterações no sistema oxitocinérgico que são altamente relevantes para o comportamento e afeto 
materno pós-parto, as fases de adaptações de vínculo e lactação também fazem parte dos eventos traumáticos, e através de um modelo feito pelo o autor foi constatado que o estresse periparto prejudica o pós-parto.

Os fatores associados ao surgimento do TEPT são o estresse adicional, baixo suporte social, falta de apoio do parceiro, entre vários outros. Já os fatores pós-natais que podem desenvolver o TEPT foram bem menos estudados. No pósparto, o bom suporte social foi considerado um grande protetor contra o desenvolvimento do TEPT (King, Mcharg \& Horsch, 2017).

Alguns dos transtornos como ansiedade, TEPT, DPP entre outros, podem ser adquiridos depois da mulher apresentar hemorragia pós-parto (HPP), pois é conhecida como uma das principais causas de mortalidades maternas em todo mundo. Algumas mulheres que também vivenciaram o HPP apresentam memórias negativas sobre o nascimento e um absurdo medo da morte (Carroll, Darly \& Begley, 2016).

Os sintomas de náuseas e vômitos de forma intensa bem no início da gestação que é caracterizado por hiperêmese gravídica (HP) é uma condição que muitas mulheres consideram traumática, sendo assim tem uma ligação com o estresse póstraumático (TEPT) e nisso as mulheres relataram que vivenciaram e se envolveram em um evento dramático e aterrorizante. Portanto o HP pode influenciar em toda a gravidez e no pós-parto causando sintomas de TEPT principalmente após o parto, também sem os devidos cuidados de forma correta dos parteiros, enfermeiros e médicos pode piorar o estado psíquico, (Kjeldgaard et al., 2018).

A cesárea de emergência (CS) é identificada como um problema psicossocial, incluindo problemas de saúde, como depressão pós parto, estresse pós-traumático, ansiedade, insatisfação materna, relação mãe-bebê, funcionamento sexual dos pais e até alimentação infantil. Esse evento (CS) pode ser estressante, provocador de ansiedade e emocionalmente perturbador para essas mulheres (Benton et al., 2019).

A perda perinatal também está associada a curto prazo e consequências de longo prazo para a saúde e bem estar, por isso muitas mulheres desenvolvem transtornos mentais como depressão e ansiedade após três meses do nascimento do bebê, e acabaram desenvolvendo o transtorno do estresse pós-traumático (TEPT). Essas mulheres apresentaram vários problemas de saúde, palpitações ou sentimentos de pânico, depressão, ansiedade, problemas de sono, lembranças do trabalho de parto ou nascimento dificuldades de concentração, dificuldades de relacionamento com marido ou parceiro e dificuldades de relacionamento com membros da família (Redshaw, Hennegan \& Henderson, 2016).

A experiência do parto apesar de ser considerada positiva, possui evidências de que pode sim ser causado pelo estresse pós-traumático em que algumas mulheres apresentam medo, impotência e ameaça durante o parto, contudo o estresse pós-traumático pode está associados a fatores pré-existentes da mulher ou de sua gravidez, condição psiquiátrica, experiências traumáticas anteriores, ansiedade, gravidez não planejada, trabalho do parto, parto prematuro, dor física, cesariana de emergência, medo para si e para o bebê, perda de controle, baixo apoio do parceiro ou até mesmo da equipe (Sentilhes et al., 2017).

Porém, pode sim haver expectativas positivas quanto ao parto e a maternidade, muitas mulheres podem não vivenciar o nascimento do seu filho de forma traumática assim obtendo resultados positivos. Mulheres podem ter esperanças e expectativas para o processo de parto que contrastam com sua experiência real (King, Mcharg \& Horshc, 2017).

\subsection{Assistência de Saúde}

Muitas mulheres desenvolvem transtornos mentais na gestação e durante o puerpério, pois nesse período vivia em situações ruins que fizesse com que surgisse alguns tramas, principalmente a depressão pós-parto devido a violência doméstica vivenciada e também traumas de violência sexual. Diante dessas situações o atendimento oferecido na Atenção Básica de Saúde (UBS) é de suma importância, pois ela será acompanhada pelo enfermeiro, um profissional que acolhe nos primeiros 
momentos da gravidez dando todo apoio, toda assistência necessária para essas mulheres que sofrem convivendo com seus traumas. Deve oferecer serviços que apoiam essas mulheres pois é de fundamental importância o acolhimento para redução dos danos causados por esses traumas (Torchalla et al., 2014).

As ações que trazem como base a promoção de saúde, tem uma abordagem centrada na família e as visitas domiciliares propostas pela Estratégia Saúde da Família parecem promissoras, já que permitem o cuidado total à mulher, considerando sua inserção familiar e social. O enfermeiro da unidade básica de saúde tem um papel fundamental, pois ele está junto a mulher durante todo o pré-natal, dando todo apoio, cuidando do seu bem estar, fazendo com que a mulher se sinta mas protegida emocionalmente, pois esses traumas causa grande agravo mental que podem ocorrer em resposta a eventos ocorrido na vida dessas mulheres (Henriques, 2015).

De acordo com Torchalla et al. (2014) as intervenções relatadas sobre traumas são distintas de intervenções traumáticas, intervenções específicas de modo que as práticas de prestação de cuidados levem em consideração a compreensão do impacto de trauma de vida, desenvolvimento e substâncias de um indivíduo. Essas práticas de intervenções são muito importantes, pois facilitam nos cuidados e no bem estar dessas mulheres que passam a conviver com esses traumas.

Optar por uma maternidade pública de referência para gestações de alto risco fetal se justifica justamente no intuito de se estimar a prevalência de TEPT entre mulheres com diferentes fatores de propensão ao agravo, inclusive características clínicas maternas e perinatais. Estudos apontam em geral que são realizados em instituições voltadas para o atendimento de gestantes de baixo risco, diante disso excluem mulheres que deram à luz bebês neomortos ou natimortos. Rápido diagnóstico e tratamento são fundamentais para melhorar a qualidade de vida da mulher e a saúde do recém-nascido, assim essa mãe poderá se sentir melhor e cuidar com bastante entusiasmo do filho (Henriques et al., 2015).

\section{Conclusão}

Neste estudo foi possível observar que os transtornos relacionados ao puerpério é uma condição patológica de alta prevalência que vem se configurando como um grande problema de saúde materna. Esse problema envolve vários aspectos, não só psicológico, como também aspectos fisiológicos e sociais.

Durante o puerpério a mãe fica vulnerável, por isso manifestam sentimentos que levam a desenvolver transtornos mentais, dentre eles, os que afetam são a depressão pós-parto (DPP), transtorno do estresse pós-traumático (TEPT) e Ansiedade, pois durante a gestação até o puerpério, ocorrem várias mudanças na vida dessas mulheres.

Nesse contexto foi possível identificar como a enfermagem está diretamente ligada no cuidado primário com essas pacientes, através da visão das próprias puérperas. Contudo é possível perceber que essas mães, mesmo com alguns desses problemas relacionados a transtornos mentais, principalmente a depressão pós-parto (DPP), muitas delas, não tiveram o suporte necessário em relação aos seus sentimentos.

Entretanto cabe ao profissional enfermeiro obstetra identificar e realizar cuidados a essas mulheres com algum tipo de transtorno, passando a ouvi-las quanto a suas necessidades e suas dúvidas, e informar também seus familiares sobre seus fatores de risco e seus principais sintomas. Nesse momento é de extrema importância o apoio da família e do profissional, pois com esse apoio a puérpera passa a ter mais confiança quanto ao seu papel de se tornar mãe. Nós buscamos compreender as evidencias científicas acerca dos determinantes psicológicos e sociais relacionados ao surgimento dos transtornos mentais no puerpério.

A maternidade, no ciclo da gravidez ao pós-parto, traz um contexto de privação muito intenso na vida das mulheres, causando vários impactos a mais, em parte temporariamente, mas também pode trazer impactos que sejam permanentes, trazendo consigo uma grande bagagem de problemas emocionais. Por isso esse público deve ser visto mais de perto por motivos relacionados a melhorar a qualidade de vida dessas mães de forma mais efetiva e acolhedora. São necessários maiores 
investimentos em cuidados e serviços de saúde como forma de diminuir a incidência de doenças emocionais e garantir a segurança e a confiança dessas mulheres afim de levar uma vida saudável e que possa vivenciar um momento de prazer e felicidade, cuidando bem da sua saúde e do seu bebê.

\section{Referências}

Abuchaim, E. S., Caldeira, N. T., Di Lucca, M. M., Varela, M., \& Silva, I. A. (2016). Depressão pós-parto e autoeficácia materna para amamentar: prevalência e associação. Acta Paul Enferm, 29(6), 664-70.

Ahmed, A., Bowen, A., \& Feng, C. X. (2017). Maternal depression in Syrian refugee women recently moved to Canada: a preliminary study. Bmc Pregnancy and Childbirth,17(1), 1-11.

Ahmed, A., Bowen, A., \& Feng, C. X., \& Muhajarine, N. (2019) Trajectories of maternal depressive and anxiety symptoms from pregnancy to five years postpartum and their prenatal predictors. BmcPregnancyAnd Childbirth,19(1), 1-10.

Airosa, S., \& Silva, I. (2013). Associação entre vinculação, ansiedade, depressão, estresse e suporte social na maternidade. Psicologia, Saúde e Doença. Lisboa, 14(1), 64-77.

Aleixo, B. A., \& Gonçalves, M. (2017) Transtorno psiquiátricos na gestação e no puerpério. Psychiatry online Brasil, 22(5).

Almeida, N. M. De C., \& Arrais, A. da R. (2016). O Pré-Natal Psicológico como programa de prevenção pós-parto. Psicologia: Ciência e Profissão, Brasília, $36(4), 847-863$

Angelini, C. R., Pacagnella, R.C., Parpinelli, M. A., Silveira, C., Andreucci, C.B., Ferreira, E.C., Santos, J. P., Zanardi, D.M., Souza, R. T., \& Caceteei, J. G. (2018) Post-Traumatic Stress Disorder And Severe maternal morbidity: is there an association?. Clinics, 73(1), 1-7.

Beck, C. T, \& Casavant, S. (2019). Synthesis of Mixed Research on Posttraumatic Stress Related to Traumatic Birth. Journal Of Obstetric, Gynecologic \& Neonatal Nursing, 48(4), 385-397.

Benton, M., Salter, A., Tape, N., Wilkinson, C., \& Turnbull, D. (2019) Women's psychosocial outcomes following an emergency caesarean section: a systematic literature review. Bmc Pregnancy and Childbirth, 19(1), 1-24.

Cantilino, A., Zambaldi, C. F., Sougey, E. B., Rennó Jr, J. (2010). Transtornos psiquiátricos no pós-parto. Archives Of Clinical Psychiatry (São Paulo), 37(6), 288-294.

Carroll, M., Daly, D., \& Begley, C. M. (2016). The prevalence of women's emotional and physical health problems following a postpartum haemorrhage: a systematic review. BmcPregnancyAndChildbirth, 16(1), 1-11.

Carvalho, G. M., Oliveira, L.R, Santos, R. V., Camiá, G. E. K., \& Soares, L. H. (2019). Transtornos mentais em puérperas: análise da produção de conhecimento nos últimos anos. Brazilian Journal of Health Review, v. 2(4), 3541-3558.

Corrêa, F. P. \& Serralha, C. A. (2015). A depressão pós-parto e a figura materna: uma análise retrospectiva e contextual. Acta Colombiana de Psicología, $18(1), 113-123$

Guedes, D. S., Souza, M., Moreira, V., \& Genestra, M. (2003). Depressão pós-parto: prevenção e conseqüências. Rev. Mal-Estar Subj, 3(2), 439-450.

Henriques, T., Moraes, C. L., Reichenheim, M. E., Azevedo, G. L., Coutinho, E. S. F., \& Figueira, I. L. V. (2015). Transtorno do estresse pós-traumático no puerpério em uma maternidade de alto risco fetal no Município do Rio de Janeiro, Brasil. Cadernos de Saúde Pública, 31(12), $2523-2534$.

King, L., Mcharg, K. M., \& Horsch, A. (2017). Testing a cognitive model to predict posttraumatic stress disorder following childbirth. Bmc Pregnancy and Childbirth, 17(1), 1-12.

Kjeldgaard, H. K., Vikanes, Å., Benth, J. Š., Junge, C., Niegel, S. G., \& Gran, M. E. (2018). The association between the degree of nausea in pregnancy and subsequent posttraumatic stress. Archives OfWomen'S Mental Health, 22(4), 493-501.

Lima, M. O. P., Tsunechiro, M. A., Bonadio, I. C., \& Murata, M. (2017). Sintomas depressivos na gestação e fatores associados: estudo longitudinal. Acta paul. enferm. [online], 30(1), 39-46.

Lopez, U., Meyer, M., Loures, V., Iselin-Chaves, I., Epiney, M., Kern, C., \& Haller, G. (2017). Post-traumatic stress disorder in parturients delivering by caesarean section and the implication of anaesthesia: a prospective cohort study. Health And Quality Of Life Outcomes,15(1), 1-13.

Maciel, L. P., Costa, J. C. C., Campos, G. M. B., Santos, N. M., Melo, R. A., \& Diniz, L. F. B. (2019). Transtorno Mental no puerpério: riscos e mecanismos de enfrentamento para a promoção de saúde. Rev. pesqui. cuid. fundam. (Online); 11(4): 1096-1102

Maeda, T. C., Parreira, B. D. M., Silva, S. R., \& Oliveira, A. C. (2014). Importância atribuída por puérperas às atividades desenvolvidas no pré-natal. Rev. Enferm. Atenção Saúde [Online],3(2):6-18.

Melo, S. B., Jordão, R. R. R., Guimarães, F. J., Perrelli, J. G. A., Cantilino, A., \& Sougey, E. B. (2018). Depressive symptoms in postpartum women at Family Health Units. Revista Brasileira de Saúde Materno Infantil, 18(1),163-169.

Ostacoli, L., Cosma, S., Evilacqua, F., Berchialla, P., Bovetti, M., Carosso, A. R., Malandrone, F., Carletto, S., \& Benedetto, C. (2020). Psychosocial factors associated with postpartum psychological distress during the Covid-19 pandemic: a cross-sectional study. Bmc Pregnancy and Childbirth, 20 (1), 1-8. 
Research, Society and Development, v. 10, n. 6, e51410616033, 2021

(CC BY 4.0) | ISSN 2525-3409 | DOI: http://dx.doi.org/10.33448/rsd-v10i6.16033

Redshaw, M., Hennegan, J. M., \& Henderson, J. (2016). Impact of holding the baby following stillbirth on maternal mental health and well-being: findings from a national survey. Bmj Open, 6(8), 1-9.

Schwengber, D. D. S., \& Piccini, C. A. (2005). A experiência da maternidade no contexto da depressão materna no final do primeiro ano da vida do bebê. Estudo de Psicologia, Campinas, 22(2), 1-14.

Sentilhes, L., Maillard, F., Brun, S., Madar, H., Merlot, B., Goffinet, F., \& Deneux-Tharaux, C. (2017). Risk factors for chronic post-traumatic stress disorder development one year after vaginal delivery: a prospective, observational study. ScientificReports, 7(1),1-9.

Soler, D. A. R. (2014). Qualidade de vida no puerpério. Faculdade de Medicina de São José do Rio Preto, 1-134.

Souza, E. A., \& Acácio, K. H. P. (2019). Acolhimento psicológico como forma interventiva no puerpério. Ciências biológicas e de saúde, 5(3), 11-23.

Souza, M. T., Silva, M. D., \& Carvalho, R. (2010). Revisão integrativa: o que é e como fazer. Einstein, 8(1)102-6.

Torchalla, I., Linden, I. A., Strehlau, V., Neilson, E. K., \& Krausz, M. (2017). Erratum to: like a lots happened with my whole childhood. Harm Reduction Journal, 14(1), 1-10.

Witteveen, A. B., Stramrood, C. A. I., Henrichs, J., Flanagan, J. C., Van Pampus, M. G., \& Olff, M. (2019). The oxytocinergic system in PTSD following traumatic childbirth: endogenous and exogenous oxytocin in the peripartum period. Archives Of Women'S Mental Health, 23(3), 317-329. 